\title{
The promise of digital mood tracking technologies: are we heading on the right track?
}

\section{THE PRESENT PROBLEM}

The impetus to develop novel ways of monitoring mood has been driven by the need to achieve a better understanding of mood disorder psychopathology. Of particular interest is the dynamic relationship between affect, mood and temperament and the particular epochs in which they reside. As depicted in figure 1, an individual's temperament lakin to personality) is thought to be relatively stable, with modulations occurring over years and decades, whereas components such as mood and affect vary more frequently and necessarily throughout an individual's life. ${ }^{1}$ Affective changes are adaptive responses to external stimuli and as such necessarily oscillate rapidly over minutes, seconds or even fractions of a second. In contrast, moods are more prolonged emotional states that tend to fluctuate over hours or days ${ }^{12}$ and follow a natural circadian pattern-the nadir usually occurring on awakening. ${ }^{3}$ The interaction between affect, mood and temperament is a dynamic process as a person's natural temperament may influence their experience of mood states, which may consequently colour their reactions to external stimuli (ie, their affective response). However, this interaction may also work in the opposite direction, such that experiencing persistent affective instability is likely to produce changes in mood, which over long periods of time may be inculcated into a person's temperament. Although this interplay between affect, mood and temperament can be readily conceptualised, assessing this relationship in practice poses a considerable challenge.

Investigating the interplay between affect, mood and temperament demands an approach that is able to detect granular affective changes in real time, while also capturing the longitudinal perspective of all these components. Historically, mood assessments have been conducted by clinicians, where clinical judgements are based on observations of the patient's current presentation, as well as subjective reports from patients themselves. ${ }^{45}$ Assessed in this manner, the components of affect, mood and temperament can be easily conflated. Additionally, reliance on interviews to obtain accurate data on mood is inherently flawed as retrospective self-reported information has been shown to be inaccurate and highly susceptible to bias. ${ }^{6}$ More recently, researchers have looked to reduce the influence of recall bias by increasing the frequency of questionnaires by employing daily diary studies or administering surveys that patients complete at the end of each week. ${ }^{7}$ However, these types of pen-and-paper surveys have been associated with disappointingly low atic search was necessary in order to capture the rapid developments in this area. 


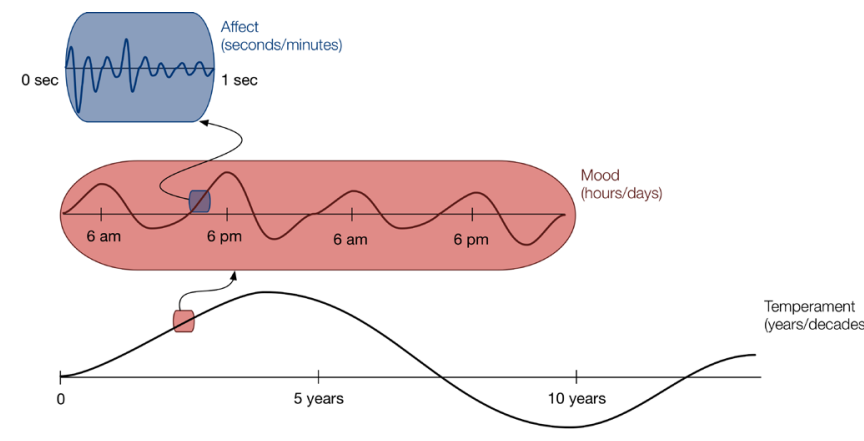

Figure 1 Varying timescales for emotional components. Affect [Top]: Momentary changes occuring over seconds and minutes. Mood [Middle]: Prolonged emotional states which can vary over hours and days. Temperament [Bottom]: Relatively stable, with modulations occuring over years and decades.

rates of participant compliance and the compilation of longitudinal data in this manner is both arduous and prone to error. ${ }^{89}$ Furthermore, although assessments are more frequent, the larger epochs of time they capture are not sufficient to capture the frequent oscillations of affect. Evidently, investigating the interplay between affect, mood and temperament requires a more sophisticated approach to tracking mood than has previously been used.

Digital technology holds great promise in enhancing our assessment of affect, mood and temperament. The increased capability, availability and affordability of technology in the form of computers, mobile phones, programmable watches and fitness tools has opened up a variety of avenues to assess mood. ${ }^{10}$ For example, the widespread dissemination of smartphones has meant that questionnaires are now able to be administered electronically and participant responses can be elicited instantaneously. ${ }^{11}$ Furthermore, smartphones are able to collect information regarding their user's call logs, physical activity levels and even their social media usage. ${ }^{12}$ Not only can this be collected in real time, improvements in storage capabilities mean that data from these devices can be easily transmitted, processed and stored with the aim of identifying longitudinal patterns. Thus, using digital technologies to assess mood is an attractive prospect for advancing mood disorder research that may help elucidate the underlying components of mood disorder psychopathology and their patterns over time.

This brief review examines the three types of data that may be of use in mood disorders research: electronic self-report, behavioural data and physiological measures.

\section{Electronic self-report}

Electronic ecological momentary assessment (EMA) is one such method adopted from the social psychology sphere that has been valuable to the field of mood disorder research. ${ }^{5}$ EMA involves the repeated administration of questionnaires that require an immediate response from participants. ${ }^{5}$ While previously being administrated through numerous paper surveys, technological advances mean that repeated questionnaires can be conveniently delivered on programmed personal digital assistants or mobile phones, thus allowing for a more instantaneous collection of data on participants' symptoms, affect, mood and behaviour at multiple points in time. ${ }^{13}$ ChronoRecord is one such program that has been widely used in research for the assessment of mania and bipolar depression. ${ }^{14}$ It involves daily self-rating by patients, and rather than completing a questionnaire on symptoms, ChronoRecord requires patients to rate their mood on a visual analogue scale ranging from 0 to 100, which has been validated against some clinical ratings, for example, the Hamilton Depression Rating Scale and the Young Mania Rating Scale. ${ }^{15}$ In addition to rating their mood state, patients are asked to describe features of their mood state, for example, reduced sleep, grandiose thoughts or hospitalisation. Over time, repeated collection of this type of data will be valuable to researchers in determining the predominant symptoms and features of bipolar depression and mania and their longitudinal patterns. This is particularly important, as the natural course of mood disorders with periodic ascents and descents into mania and depression are often preceded by signs and symptoms that herald the onset of a full-blown episode. ${ }^{16}$ These are often referred to as early warning signs (EWS). Repeated momentary assessments, administered through mobile phones, could help better understand EWS and reveal how environmental triggers may interact with other factors and precipitate the onset of depression or mania which is likely to inform the development of relapse prevention strategies. $^{10}$

The adoption of electronic EMA by researchers has already been valuable in broadening the scope of mood disorder research and deepening our understanding of dynamic processes. For example, Ebner-Priemer and Kuo ${ }^{17}$ conducted an EMA study where patients were digitally reminded to rate their affect every 10 to 20 min over a duration of 24 hours and found that patients with borderline personality disorder (BPD) reported shorter times between affect fluctuations in comparison with healthy controls. Although affective instability is already widely recognised as a core feature of patients with BPD, using digital technology to assess affect in such short increments revealed that $48 \%$ of extreme mood changes occurred within a 15 min time period. Information this detailed simply could not have been gathered using traditional surveys or clinical interviews.

Despite the practical advantages and research benefits that have already been seen in adopting digital EMA methods, there are some inherent limitations as to 'what' is being assessed. First, although EMA attempts to ameliorate the problem of retrospective memory bias when recalling symptoms, there is still an element of recall involved in electronic EMA methods depending on the epoch of time being sampled. ${ }^{13}$ In order to reduce recall bias further, assessments would need to be far more frequent. However, repeatedly administering the same survey and requiring an immediate response could quickly become burdensome and intrusive and may influence the accuracy of patient responses. ${ }^{8}$ Thus, if these surveys are to be used for gaining a longitudinal perspective on patients with mood disorders over years of assessment, researchers will need to find the balance of collecting precise and timely information in a way that is amenable to patients over the long term. The second limitation of EMA, more broadly, is that it is still subject to patient self-report. While clinical interviews are based on both verbal reports from patients and objective clinical observations, EMA methods rely solely on patient accounts and self-ratings that are necessarily subjective and vulnerable to personal bias. Overcoming this issue of subjectivity may require us to do more than just improving the method of collecting data by using digital surveys and rating systems, and might entail the development of more creative and novel ways of inferring mood apart from self-reported information.

\section{THE FUTURE}

Continuous, real-time monitoring of the physiological, biological and behavioural symptoms of patients with mood disorders may provide the objective measures needed to overcome the limitations of EMA and the subjective nature of self-report. Until recently technological restrictions and associated costs have prevented the remote acquisition of objective behavioural and physiological data, leaving many unanswered questions regarding the biological mechanisms underpinning mood disorders. Modern developments such as smartphones with built-in or external sensors have enabled the remote capture of biomarker data. While mobile apps and programs designed to record electronic EMA and self-report mood ratings are increasingly common, ${ }^{18}{ }^{19}$ there are relatively few empirically supported objective tools that validate self-reported data and measure potential biomarkers associated with mood disorders. 


\section{Behavioural data}

Several studies have used smartphone sensors to monitor behavioural patterns and levels of physical activity in an attempt to identify subsyndromal mood symptoms in real time. ${ }^{2021}$ Mobile sensors are being used to passively collect trace data from GPS, call logs and phone activity to track behaviours such as mood, fatigue, physical isolation and social activity. Exploratory data have revealed that GPS trace data is strongly correlated with depressive symptom severity. ${ }^{22}$ GPS data provides behavioural information by means of indicating a patient's travel patterns. During both depressive and manic states, travel and movement patterns tend to change (eg, depressed people tend to move around less and travel less). Several studies have demonstrated that monitoring physical activity through smartphone GPS data correlates with changes in emotional states. ${ }^{23-25}$ Real-time behavioural data have been used as a proxy of mood, however, it is not clear whether physical activity is a precursor of mood changes or a consequence. Similar to GPS data, data extracted from phone usage activity and call logs has been used to indicate social interactions or level of social activity, and therefore significant changes in activity can be used to predict mood states. Several studies have monitored phone call and SMS logs (for frequency, duration, and incoming/ outgoing interactions) and data from apps or online social networks (eg, Facebook, Twitter and emails) and have found that usage patterns and language choices can be used to infer mood changes. ${ }^{26-28}$ The real-time and longitudinal data collected from these devices may provide some necessary information for investigating the interaction between affect, mood and temperament.

Voice and speech analysis is another form of behavioural data that can be easily recorded by biosensors in smartphone devices. Speech analysis has been investigated as a passive method of examining mental state as psychological states modulate the production of speech. ${ }^{29}$ Analysis of vocal cues (eg, content, prosody, voice quality and tone of voice) has been correlated with emotional states. ${ }^{30} 31$ Several studies investigating smartphone-based systems proposed that audio, accelerometer and other speech data can be used to classify mood states in bipolar disorder patients. ${ }^{24233}$ In particular, Abdullah et a ${ }^{32}$ found that they could identify mood states in bipolar patients with at least $80 \%$ accuracy by using data collected from the patient's day-to-day phone calls. However, similar to self-report data this type of data can be susceptible to recall bias. That is, an individual could be recalling past emotional events when speaking on the phone and therefore vocal cues may not be reflective of their current emotional state.

\section{Wearable physiological data}

To enhance our understanding even further and to overcome some of the limitations of behavioural data collection, researchers in this field have shifted their focus to utilising the emerging trend of wireless wearable biosensors. Wearable biosensors allow for the instantaneous collection of physiological data such as neural activity, heart rate variability (HRV) and skin conductance to act as objective identifiers of mood states. This research follows the current high demand for mobile health and fitness devices or 'wearables' such as fitness watches.

The collection of instantaneous physiological data may be an immensely important step in determining the mechanistic foundation of mood. For example, whether these physiological changes are a consequence of mood changes or whether they are the cause of such variability. A recent meta-analysi ${ }^{34}$ has demonstrated that there is a consistent reduction in HRV in patients with psychiatric disorders compared with control patients. HRV can be used as a psycho-physiological biomarker because it mirrors the variability of heart rate in response to affective and cognitive states. ${ }^{35}$ Several groups of researchers have developed various 'wearable' monitoring devices to try to capture real-time HRV data in order to improve knowledge of bipolar disorder by identifying potential biomarkers. Valenza et $a^{\beta 6}$ have developed a wearable shirt that integrates electrodes

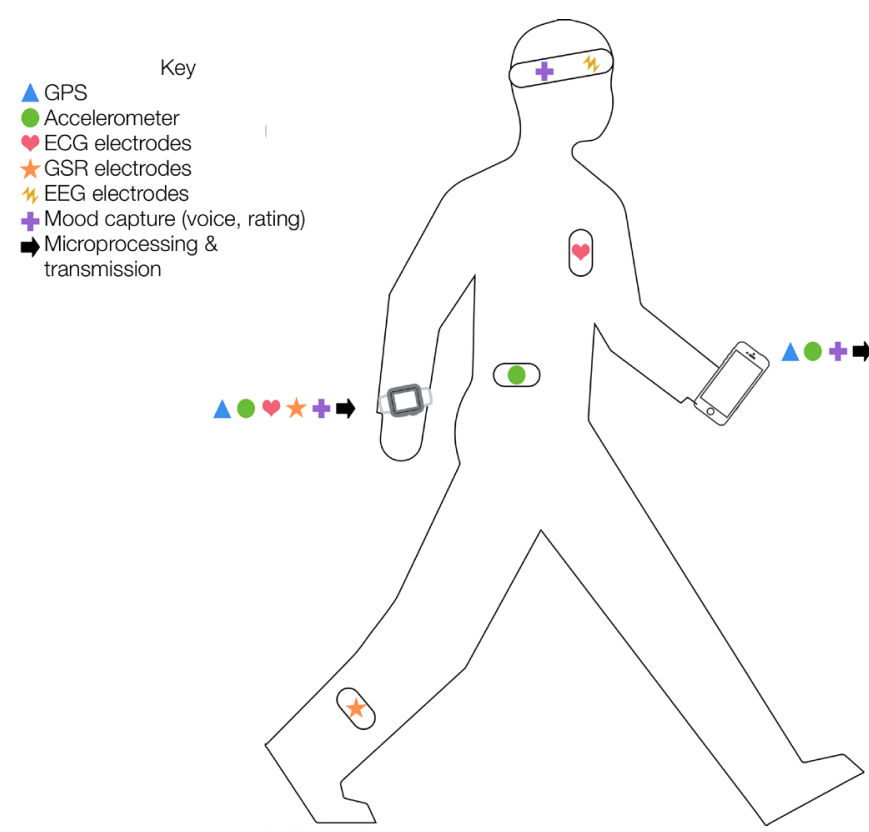

Figure 2 Portable and wearable devices. Various electrodes and other sensors can be attached to the body or embedded into everyday clothing/accessories. Processing and transmission of data can be performed using devices such as smartwatches and smartphones.

and sensors into the fabric to instantaneously record HRV and detect depressive, mixed or hypomanic mood states in bipolar disorder patients. Roh et a ${ }^{37}$ have created a low-powered, light-weight headband monitoring system with embedded electrodes to record EEG and HRV to classify mental states in real time. Skin conductance or electrodermal activity is a biomarker of arousal and has been used to validate self-reported arousal levels. Novel systems for measuring electrodermal activity have been developed by Kappeler-Setz et $a^{38}$ and they have trialled sensor systems in shoes and socks, revealing that recording electrodermal activity through patients' feet may be a suitable way to record emotional states and stress levels in bipolar patients (see figure 2).

\section{CONSIDERATIONS AND LIMITATIONS}

While instantaneously measuring and analysing biomarkers has advanced research into the physiological mechanisms underpinning mood state, there still remain some serious logistical, technological and ethical considerations within this field (for pros and cons of each type of digital monitoring device, see table 1). Primarily, smartphone and external biosensors continuously collect large amounts of data, meaning any analytic approaches must be suitable for 'big data' and analysis would require a large amount of technical and computational expertise. Maintaining the quality of the data limits the execution of research and clinical use but also increases the personnel and direct costs. Theinitial development and maintenance of biosensor devices and software can be extremely costly. Therefore, complicated biosensor systems may not currently be realistic in a large-scale, long-term clinical setting. Additionally, due to the volume and type of data collected (ie, sensitive personal, behavioural and physiological information), data security, privacy and confidentiality issues are a major concern which is compounded by the issue of 'ownership' over the data. Comprehensive technical consultation and intensive management is essential when developing and using digital monitoring technologies for both research and clinical purposes.

Additionally, there are concerns regarding patient adherence to both active and passive digital monitoring programs over the long term, especially when patients experience periods of remission. While this is an 
Table 1 Pros and cons of current digital mood tracking techniques

\begin{tabular}{|c|c|c|}
\hline Technology & Pros & Cons \\
\hline \multicolumn{3}{|l|}{ Electronic self-report } \\
\hline Digital EMA & $\begin{array}{l}\text { Can easily administer questionnaires and elicit immediate } \\
\text { responses from participants } \\
\text { Can use validated rating scales } \\
\text { Increased compliance compared with pen-and-paper surveys }\end{array}$ & $\begin{array}{l}\text { Repeated questionnaires can become burdensome to } \\
\text { participants } \\
\text { - Frequency of EMA can be intrusive } \\
\text { Reliance on self-reported information }\end{array}$ \\
\hline \multicolumn{3}{|l|}{ Behavioural monitoring } \\
\hline \multicolumn{3}{|l|}{ GPS } \\
\hline Location and behaviour tracking & $\begin{array}{l}\text { Basic technology embedded in normal smartphone devices } \\
\text { (therefore easily accessible and cheap) }\end{array}$ & $\begin{array}{l}\text { Location and movement does not immediately translate to } \\
\text { social interaction }\end{array}$ \\
\hline \multicolumn{3}{|l|}{ Phone activity } \\
\hline $\begin{array}{l}\text { Internet usage (including social } \\
\text { media) }\end{array}$ & $\begin{array}{l}\text { Data are easily recorded } \\
\text { Non-invasive } \\
\text { Longitudinal monitoring }\end{array}$ & $\begin{array}{l}\text { Does not necessarily reflect social activity (not face-to-face } \\
\text { interactions) } \\
\text { Connectivity issues }\end{array}$ \\
\hline Calls logs & $\begin{array}{l}\text { Data are easily recorded } \\
\text { Non-invasive } \\
\text { Longitudinal monitoring }\end{array}$ & $\begin{array}{l}\text { Individuals are typically very unpredictable and variable - hard } \\
\text { to determine what may be abnormal for each individual }\end{array}$ \\
\hline Speech analysis & $\begin{array}{l}\text { Data are easily recorded } \\
\text { Non-invasive } \\
\text { Longitudinal monitoring }\end{array}$ & $\begin{array}{l}\text { May be subject to recall bias - voice could be modified due to } \\
\text { recalling a previous emotional time } \\
\text { Advanced programs analysis required }\end{array}$ \\
\hline \multicolumn{3}{|l|}{ Physiological monitoring } \\
\hline \multicolumn{3}{|l|}{ Wearables } \\
\hline EEG (eg, headband or hat) & - Unparalleled access to real-time neural activity & $\begin{array}{l}\text { Non-compliance, not feasible to wear continuously } \\
\text { Advanced programs and analysis required }\end{array}$ \\
\hline HRV (eg, shirt or multisensor watch) & $\begin{array}{l}\text { HRV is a empirically supported psycho-physiological } \\
\text { biomarker- immediate HRV feedback is important for research } \\
\text { and also for monitoring clinical care }\end{array}$ & $\begin{array}{l}\text { Expensive to create electrodes embedded into shirts } \\
\text { Not feasible to always wear HRV monitoring shirt } \\
\text { Not very accurate recording from multisensor watches } \\
\text { Advanced programs and analysis required }\end{array}$ \\
\hline Skin conductance (eg, socks) & - Easily assess arousal and stress levels & $\begin{array}{l}\text { Expensive to create and monitor } \\
\text { Not feasible to wear all of the time } \\
\text { Advanced programs and analysis required }\end{array}$ \\
\hline
\end{tabular}

EMA, ecological momentary assessment; GPS, global positioning system; HRV, heart rate variability.

ongoing concern, there has been promising evidence supporting the feasibility of longitudinal use of such digital mood tracking methods. For example, digital self-report studies using clinical populations by Bopp et $a l^{8}$ and Tsanas et $a l^{39}$ have reported compliance levels of $75 \%$ and $>80 \%$ respectively, suggesting that digital mood tracking may be readily incorporated as part of patient care in the future. While adherence seems to be an issue that can be overcome, another potential ethical issue that requires further investigation is the concern that repeatedly asking depressed patients to rate their mood could increase the risk of patients focusing on their depression. Further research is required to establish whether this is a valid ethical concern.

It must be acknowledged that all of the current biosensor research is based on behavioural inferences, such that data collected from digital biosensors can be mapped on to psychological variables (eg, social activity, physical activity and mood states). ${ }^{40}$ It is therefore necessary to appraise these inferences by examining the validity and reliability of the measures. Both the construct and external validity of biosensor and behavioural data must be evaluated to determine whether the objective measures correlate with the self-report measures and importantly whether the objective measures capture the intended processes. It is possible that physiological and behavioural data may only capture the precursors to emotional state changes or the consequences of change without tapping into the key construct itself. Each different biosensor or device may be capturing a different aspect of affect, mood or temperament, or they may not be capturing the core component of emotional states at all. Currently, at each step along the pathway from pathophysiology to formulation, information is being lost, resulting in our lack of certainty and knowledge surrounding objective measures of mood (see figure 3). The proximity of source is currently one of the major barriers to effective objective digital mood monitoring. For example, the closer the technological device is to capturing the exact physiological change then the more accurate the formulation will be. Subsequently, current studies may be underestimating or overestimating certain behaviours due to limitations in the technology, variations in the data and further loss of information during analysis. It is important for future research to elucidate these psychometric considerations in developing suitable behavioural measures.

In order to overcome the limitations of available digital mood tracking technologies, further research is necessary to first confirm which valid objective measures to target and to consequently refine the technology to be able to capture that construct as closely as possible.

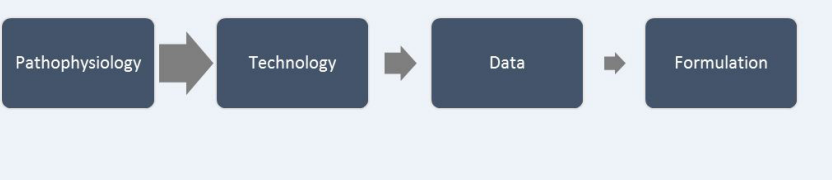

Figure 3 Steps and filters in the translation of physiological information to clinical formulation. Pathophysiology of mood occurs and current technological devices such as smartphones and biosensors record certain behavioural and physiological changes. The recorded data is then analysed, where more of the original information is lost. This analysis is used for formulation and understanding mood. The majority of the information is lost by the formulation phase. 


\section{POTENTIAL DIRECTIONS}

In addition to extending and deepening our understanding of mood disorders in the research sphere, digital mood tracking technologies have the potential to enhance the clinical management of mood disorders as the repeated self-report and biosensor data they produce can give clinicians a more comprehensive picture of a patient's illness pattern when making decisions regarding treatment. ${ }^{20}$ Digital technologies could also be used to provide alerts to patients or clinicians when unusual symptom clusters or EWS are logged, and could even prompt patients to adjust their treatment or engage in a psychosocial intervention prior to the onset of an episode. Passive monitoring could also be useful in this regard, as Pejovic et a $\left.\right|^{41}$ used GPS data from participants' mobile sensors to trigger a self-report questionnaire which generated relevant context-contingent questions. ${ }^{40}$ This type of contextual data collection is one example of how further technological advancement could be extremely useful in detecting EWS. However when developing these mood tracking technologies and determining their capabilities, it is important to draw on the perspectives of clinicians in order to ensure that these novel methods are actually targeting unmet clinical needs.

There has already been a modest clinical uptake of digital self-report programs, for example, MoodScope ${ }^{42}$ which has the ability to send data to family members or healthcare professionals. However, many other programs are yet to be administered systematically outside of research studies. ${ }^{43}$ To date, there has been limited clinical uptake of passive mood monitoring; however, the 'iSee' initiative developed by Michigan State University ${ }^{44}$ aims to use students' behavioural and GPS data to enable counselling services to engage with target populations more effectively. Thus, digital self-report programs and passive monitoring have potential to greatly enhance the management of mood disorders in the clinical sphere. However, prior to the widespread adoption of these digital tracking technologies in clinical practice, it is imperative that clinicians are consulted as they may be responsible for monitoring, interpreting and acting on the real-time data they receive.

\section{CONCLUSION}

Smartphone and other mobile devices, such as wearables, have become interlaced with our daily lives. It has opened a window to investigate real-time human behaviour with unprecedented granularity. Access to such data has already provided researchers with new insights into mood tracking and the behavioural underpinnings of mood disorders. With continued multidisciplinary collaboration (eg, with software engineers and computer scientists) and ever-increasing technological advances, the use of smartphone and biosensor tracking will not only provide a deeper understanding of mood states and behaviour, but can also improve clinical care for patients with mood disorder.

Competing interests None declared.

Provenance and peer review Not commissioned; externally peer reviewed doi:10.1136/eb-2017-102757

Received 15 June 2017; Revised 31 July 2017; Accepted 9 August 2017

\section{REFERENCES}

1. Gray EK, Watson D, Payne R, et al. Emotion, mood, and temperament: Similarities, differences, and a synthesis. Emotions at work: Theory, research and applications for management. Chichester: Wiley, 2001:21-43.

2. Wilhelm P, Schoebi D. Assessing mood in daily life. Eur J Psychol Assess 2007;23:258-67.

3. Boivin DB, Czeisler CA, Dijk DJ, et al. Complex interaction of the sleep-wake cycle and circadian phase modulates mood in healthy subjects. Arch Gen Psychiatry 1997;54:145-52.

4. Ebner-Priemer UW, Trull TJ. Ecological momentary assessment of mood disorders and mood dysregulation. Psychol Assess 2009;21:463-75.

5. Moskowitz DS, Young SN. Ecological momentary assessment: what it is and why it is a method of the future in clinical psychopharmacology. J Psychiatry Neurosci 2006; $\mathbf{3 1 : 1 3}$
6. Yasui-Furukori N, Nakamura K. Bipolar disorder recurrence prevention using selfmonitoring daily mood charts: case reports from a 5 year period. Neuropsychiatr Dis Treat 2017;13:733-6.

7. Cranford JA, Shrout PE, lida M, et al. A procedure for evaluating sensitivity to withinperson change: can mood measures in diary studies detect change reliably? Pers Soc Psychol Bull 2006;32:917-29.

8. Bopp JM, Miklowitz DJ, Goodwin GM, et al. The longitudinal course of bipolar disorder as revealed through weekly text messaging: a feasibility study. Bipolar Disord 2010;12:327-34.

9. Bauer M, Grof P, Gyulai L, et al. Using technology to improve longitudinal studies: self-reporting with ChronoRecord in bipolar disorder. Bipolar Disord 2004;6:67-74.

10. Wenze SJ, Miller IW. Use of ecological momentary assessment in mood disorders research. Clin Psychol Rev 2010;30:794-804.

11. Areàn PA, Hoa Ly $K$, Andersson G. Mobile technology for mental health assessment. Dialogues Clin Neurosci 2016;18:163.

12. Heron KE, Smyth JM. Ecological momentary interventions: incorporating mobile technology into psychosocial and health behaviour treatments. Br J Health Psychol 2010;15:1-39.

13. Stone AA, Shiffman S. Capturing momentary, self-report data: a proposal for reporting guidelines. Ann Behav Med 2002;24:236-43.

14. Whybrow PC, Grof P, Gyulai L, et al. The electronic assessment of the longitudinal course of bipolar disorder: the ChronoRecord software. Pharmacopsychiatry 2003;36(Suppl 3):244-9.

15. Bauer M, Wilson T, Neuhaus K, et al. Self-reporting software for bipolar disorder: validation of ChronoRecord by patients with mania. Psychiatry Res 2008;159:359-66.

16. Morriss RK, Faizal MA, Jones AP, et al. Interventions for helping people recognise early signs of recurrence in bipolar disorder. Cochrane Database Syst Rev 2007:CD004854.

17. Ebner-Priemer UW, Kuo J, Kleindienst N, et al. State affective instability in borderline personality disorder assessed by ambulatory monitoring. Psychol Med 2007;37:961-70.

18. Faurholt-Jepsen M, Ritz C, Frost M, et al. Mood instability in bipolar disorder type I versus type II-continuous daily electronic self-monitoring of illness activity using smartphones. J Affect Disord 2015;186:342-9.

19. Torous J, Staples P, Shanahan M, et al. Utilizing a Personal Smartphone Custom App to Assess the Patient Health Questionnaire-9 (PHO-9) Depressive Symptoms in Patients With Major Depressive Disorder. JMIR Ment Health 2015;2:e8.

20. Place S, Blanch-Hartigan D, Rubin C, et al. Behavioral Indicators on a Mobile Sensing Platform Predict Clinically Validated Psychiatric Symptoms of Mood and Anxiety Disorders. J Med Internet Res 2017;19:e75.

21. Saeb S, Zhang M, Karr CJ, et al. Mobile Phone Sensor Correlates of Depressive Symptom Severity in Daily-Life Behavior: An Exploratory Study. J Med Internet Res 2015;17:e175

22. Saeb S, Lattie EG, Schueller SM, et al. The relationship between mobile phone location sensor data and depressive symptom severity. PeerJ 2016:4:e2537.

23. Ben-Zeev D, Scherer EA, Wang R, et al. Next-generation psychiatric assessment: Using smartphone sensors to monitor behavior and mental health. Psychiatr Rehabil J 2015;38:218-26.

24. Grünerbl A, Muaremi A, Osmani V, et al. Smartphone-based recognition of states and state changes in bipolar disorder patients. IEEE J Biomed Health Inform 2015;19:140-8.

25. Palmius N, Tsanas A, Saunders KEA, et al. Detecting bipolar depression from geographic location data. IEEE Trans Biomed Eng 2017;64:1761-71.

26. Alvarez-Lozano J, Osmani V, Mayora 0, eds. Tell me your apps and I will tell you your mood: correlation of apps usage with bipolar disorder state. Proceedings of the 7th International Conference on PErvasive Technologies Related to Assistive Environments. ACM, 2014.

27. De Choudhury M, Gamon M, Counts S, eds. Predicting Depression via Social Media. ICWSM, 2013

28. Katikalapudi R, Chellappan S, Montgomery F, et al. Associating depressive symptoms in college students with internet usage using real Internet data. IEEE Technology and Society Magazine 2012;31:73-80.

29. Hopkins CS, Ratley RJ, Benincasa DS, eds. Evaluation of voice stress analysis technology. System Sciences, 2005 HICSS'05 Proceedings of the 38th Annual Hawaii International Conference on. IEEE, 2005.

30. Chang K-h, Fisher D, Canny J, eds. How's my mood and stress?: an efficient speech analysis library for unobtrusive monitoring on mobile phones. Proceedings of the 6th International Conference on Body Area Networks. ICST (Institute for Computer Sciences, Social-Informatics and Telecommunications Engineering), 2011

31. Trevino AC, Quatieri TF, Malyska N. Phonologically-based biomarkers for major depressive disorder. EURASIP J Adv Signal Process 2011;2011:42.

32. Abdullah $\mathbf{S}$, Matthews $M$, Frank E, et al. Automatic detection of social rhythms in bipolar disorder. J Am Med Inform Assoc 2016;23:538-43.

33. Muaremi A, Gravenhorst F, Grünerbl A, eds. Assessing bipolar episodes using speech cues derived from phone calls. International Symposium on Pervasive Computing Paradigms for Mental Health. Springer, 2014. 
34. Alvares GA, Quintana DS, Hickie IB, et al. Autonomic nervous system dysfunction in psychiatric disorders and the impact of psychotropic medications: a systematic review and meta-analysis. J Psychiatry Neurosci 2016;41:89-104.

35. Berntson GG, Cacioppo JT. Heart rate variability: Stress and psychiatric conditions. Dynamic electrocardiography, 2004:57-64.

36. Valenza G, Nardelli M, Lanatà A, et al. Wearable monitoring for mood recognition in bipolar disorder based on history-dependent long-term heart rate variability analysis. IEEE J Biomed Health Inform 2014;18:1625-35.

37. Roh T, Bong K, Hong S, eds. Wearable mental-health monitoring platform with independent component analysis and nonlinear chaotic analysis. Engineering in Medicine and Biology Society (EMBC), 2012 Annual International Conference of the IEEE. IEEE, 2012

38. Kappeler-Setz C, Gravenhorst F, Schumm J, et al. Towards long term monitoring of electrodermal activity in daily life. Pers Ubiquitous Comput 2013;17:261-71.

39. Tsanas A, Saunders KE, Bilderbeck AC, et al. Daily longitudinal self-monitoring of mood variability in bipolar disorder and borderline personality disorder. J Affect Disord 2016:205:225-33
40. Harari GM, Lane ND, Wang R, et al. Using Smartphones to Collect Behavioral Data in Psychological Science: Opportunities, Practical Considerations, and Challenges. Perspect Psychol Sci 2016;11:838-54.

41. Pejovic V, Lathia N, Mascolo C, et al. Mobile-based experience sampling for behaviour research. Emotions and Personality in Personalized Services: Springer 2016:141-61.

42. Drake G, Csipke E, Wykes T. Assessing your mood online: acceptability and use of Moodscope. Psychol Med 2013;43:1455-64.

43. Isometsä E. Mood Zoom could be a promising tool for daily mood variability monitoring, potentially differentiating bipolar from borderline patients. Evid Based Ment Health 2017;20:e9.

44. Zhang M, Mohr D, Meng J. Helping universities combat depression with mobile technology [Internet]. The Conversation 2017 http://theconversation.com/ helping-universities-combat-depression-with-mobile-technology-67033 (cited 27 July 2017). 